\title{
Structure of Low-Loss EELS in Rhenium
}

\author{
R.W. Carpenter and Andrew V.G. Chizmeshya
}

Center for Solid State Science, Arizona State University, Tempe AZ, 85287-1704 U.S.A.

The fine structure of ionization edges in EELS is typically used to study both the electronic structure and elemental composition of materials. The interpretation and analysis is greatly simplified when there is no energetic overlap with neighboring energy loss features (e.g., other ionization edges). This is the case for core-loss EELS features of common elements. The low-loss spectra $(<60 \mathrm{eV})$ of heavy elements in the transition metal sequence possess a very rich constellation of spectral features characterized by: (i) ionization edges arising from transitions between relatively shallow semi-core states and valence (VEELS) and/or conduction band states and (ii) plasmon and plasmon-like features. The complexity arising from the overlap of these features has made a detailed understanding difficult to attain.

In this paper we apply first principles density functional theory to simulate the low-loss EELS features using the full potential linearized augmented plane wave (FPLAPW) method [1,2]. A related effort was recently reported in these abstracts [3] for the case of Ag, where it was shown that the essential features in the low-loss spectrum emerge from a careful calculation of the dielectric function. In principle the dielectric function of a solid embodies all electronic transitions, including those originating from the promotion of core electrons. In practice the dielectric function obtained from band structure calculations explicitly includes only contributions involving valence and conduction band states. The semi-core/valence separation implicit in this treatment then requires the core and core-like (e.g., semi-core in origin) contributions to be calculated separately. In the following we follow this procedure for hexagonal close packed Rhenium. The shallow $\mathrm{O}_{2,3}$ and $\mathrm{N}_{6,7}$ semi-core energy loss features associated with transitions from $5 \mathrm{p}$ and $4 \mathrm{f}$ electrons, respectively, are computed following the general method of Nelhiebel et al. [4], while lower energy spectral features are derived from the loss function $\operatorname{Im}(-1 / \varepsilon)$, which we obtain explicitly from the dielectric function $[2]$.

Figure 2 illustrates that these contributions, when taken together, give a good account of the experimental low-loss spectrum. The origin of certain prominent features labeled on the theoretical spectrum can be simply understood in terms of the density of states, which is shown in the adjoining right panel. For example, feature "d" accrues from a broad range of dipolar inter-band transitions between the d-symmetry (valence) and p-symmetry(conduction) band states. The EELS cross sections for $\mathrm{O}_{2,3}$ and $\mathrm{N}_{6,7}$ core loss edges were simulated using the same converged band states used in the dielectric and loss function [4]. These calculations involve transitions from $5 \mathrm{p}$ and $4 \mathrm{f}$ atomic states, which are probably best characterized as shallow semi-core like. The basis set and muffin-tin radii were judiciously chosen to avoid non-orthogonality errors associated with wavefunction leakage. The $\mathrm{O}_{2,3}$ and $\mathrm{N}_{6,7}$ core-loss edge onsets were located at $41 \mathrm{eV}$ and $49 \mathrm{eV}$, respectively.

[1] K. Schwarz, P. Blaha and G.K.H. Madsen, Computer Physics Communications 147, 71 (2002).

[2] "Exciting" FP-LAPW program, http://www.exciting.physics.at/ (2004).

[3] V.J. Keast, Microsc. Microanal. 10 (suppl. 2), 854 (2004). 
[4] M. Nelhiebel, P.-H. Louf, P. Schattschneider, P. Blaha, K. Schwarz, and B. Jouffrey, Phys. Rev. B 59, 12807 (1999).
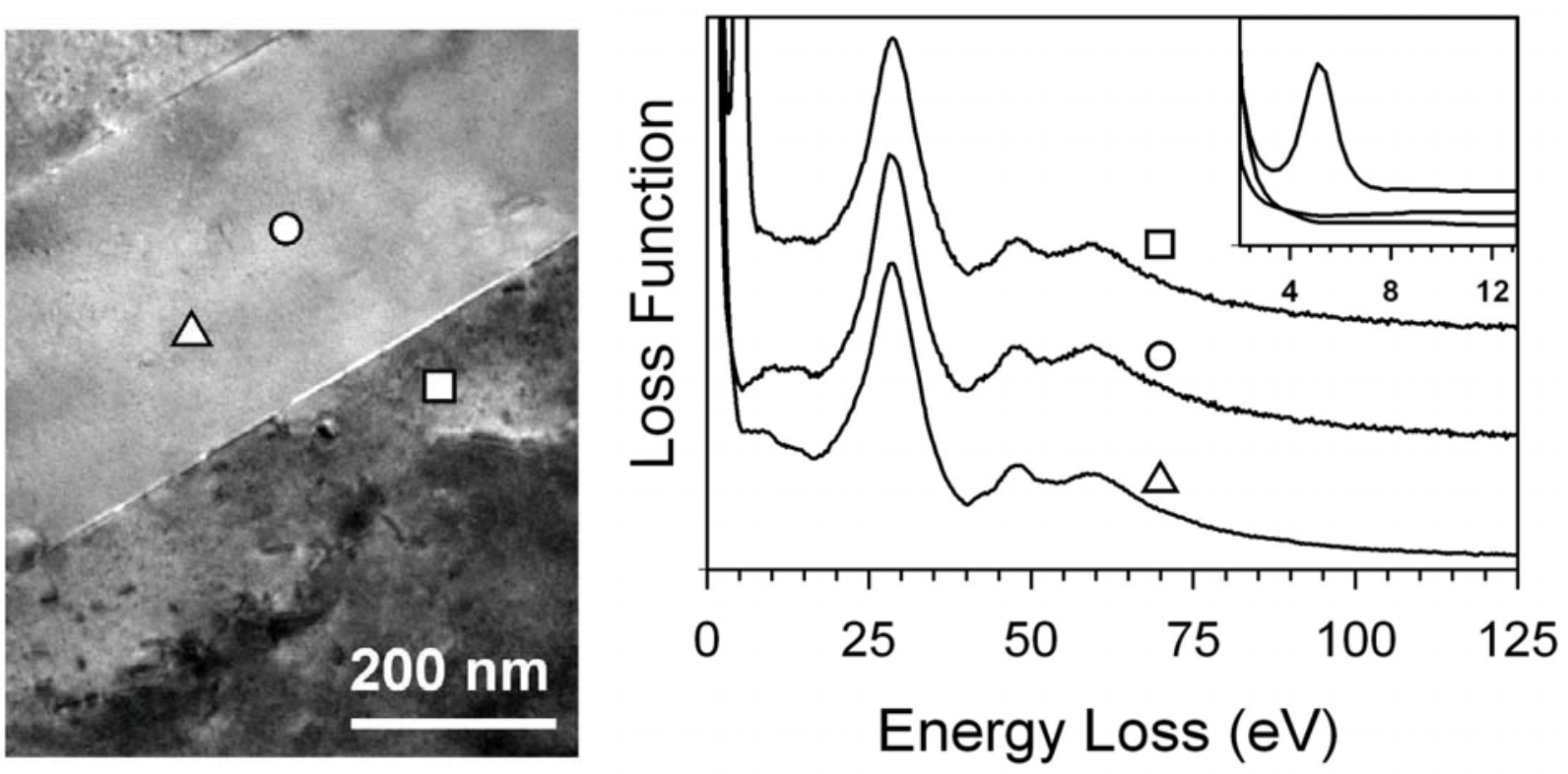

Figure 1: Left panel: Micrograph of the twin region in the Rhenium sample. Circle and square indicate locations within the twin region (circle) and outside the twin region (square) where analysis was performed. Right panel: Low-loss EELS corresponding to the three collection regions indicated by the triangle, circle and square in the micrograph (left).
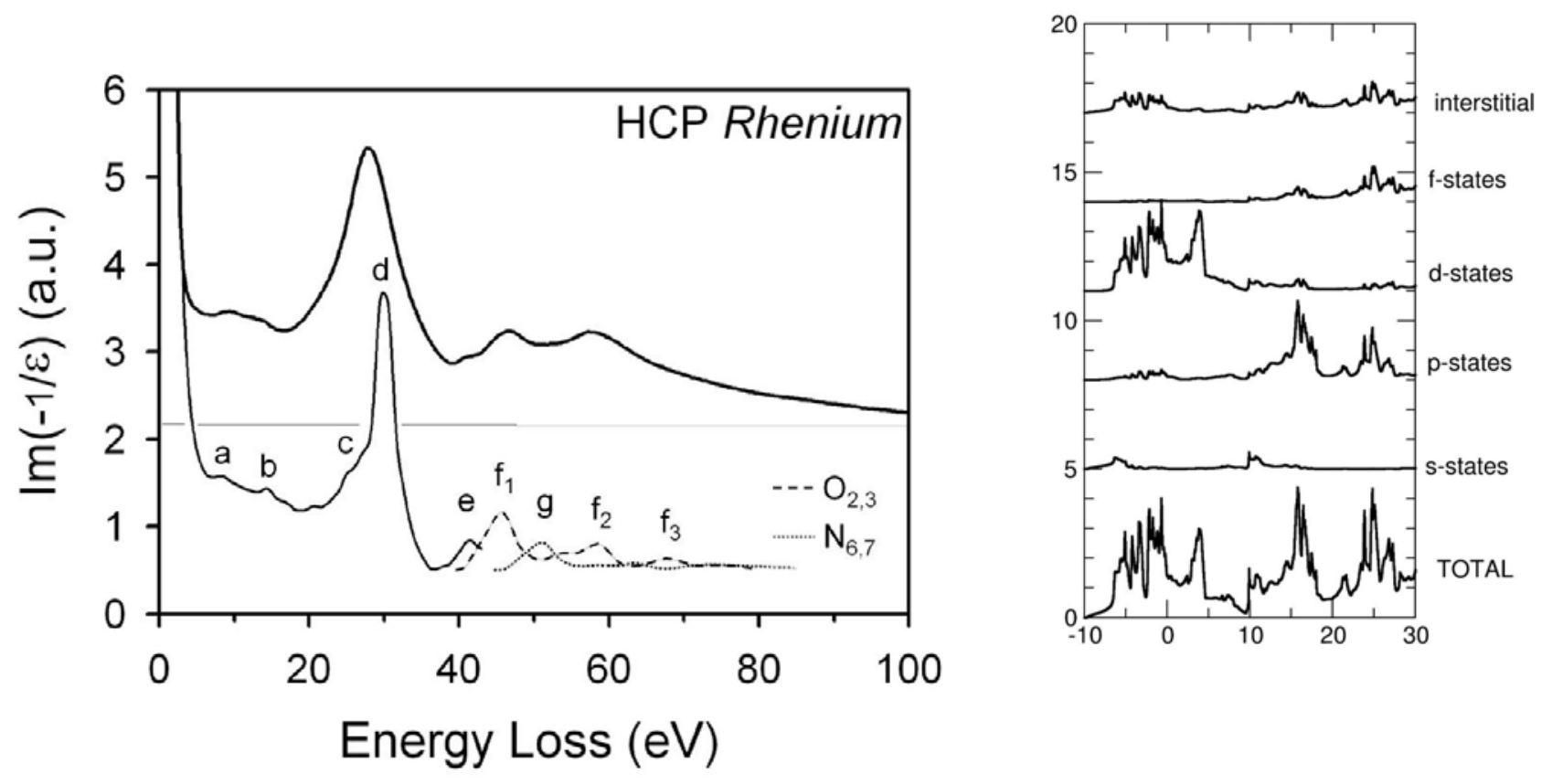

Figure 2: Left panel: Experimental (bold, top trace) and calculated (bottom traces) energy loss functions for rhenium. Right panel: Density of states of Rhenium (bottom trace), including angular momentum decomposition. 